\title{
Extracción etanólica de aceite de semillas de Moringa oleifera
}

\author{
Ethanolic extraction of oil from Moringa oleifera seeds
}

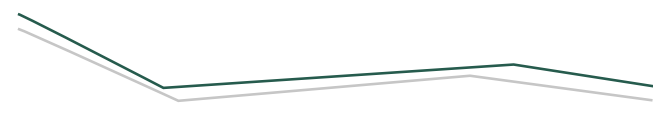

Danger Tabio García*, Claudia Espinosa Acosta*, Yosvany Díaz Domínguez*, Maylin Rondón Macias*, Elina Fernández Santana*, Ramón Piloto-Rodríguez**®

Tabio García, D., Espinosa Acosta, C., Díaz Domínguez, Y., Rondón Macias, M. Fernández Santana, E., \& Piloto-Rodríguez, R. (2018). Extracción etanólica de aceite de semillas de Moringa oleífera. Investigación y Ciencia de la Universidad Autónoma de Aguascalientes, 26(74), 32-38.
\end{abstract}

\section{RESUMEN}

La Moringa oleifera es altamente valorada a nivel mundial debido a sus propiedades. Una de las partes aprovechables del árbol son las semillas. En el presente trabajo se desarrolló la extracción de aceite mediante un extractor Soxhlet a partir de semillas de Moringa oleifera Lam. variedad Plain de origen cubano. El porcentaje de extracción se estableció como variable respuesta en el diseño de experimentos. Los factores temperatura de extracción y relación soluto-solvente influyeron de manera significativa en la variable respuesta, teniendo asociados valores de probabilidades menores que 0,05 . El modelo matemático obtenido explicó $94,03 \%$ de la variabilidad en la variable respuesta. La mejor condición para la extracción de aceite se alcanzó operando a una relación solutosolvente 1:6 y a una temperatura de extracción de $65^{\circ} \mathrm{C}$.

Palabras clave: Moringa oleifera; semillas; aceite; secado; cinética; extracción con solventes.

Keywords: Moringa oleifera; seeds; oil; drying; kinetics; solvent extraction.

\section{Recibido: 11 de julio de 2017, aceptado: 20 de marzo de 2018}

* Departamento de Ingeniería Química, Universidad Tecnológica de la Habana "José Antonio Echeverría". Calle 114, No. 11901. e/ Ciclovía y Rotonda, Marianao, C. P. 19390, La Habana, Cuba. Correo electrónico: dangerss@quimica.cujae.edu.cu; cespinosaa@quimica.cujae. edu.cu;ydiaz@quimica.cujae.edu.cu; maylin91@quimica.cujae.edu. cu; elina@quimica.cujae.edu.cu

** Centro de Estudios de Tecnologías Energéticas Renovables, Universidad Tecnológica de la Habana "José Antonio Echeverría". Calle 114 No. 11901. e/ Ciclovía y Rotonda, Marianao, C. P. 19390, La Habana, Cuba. Correo electrónico: rpiloto@ceter.cujae.edu.cu
ABSTRACT

Moringa oleifera is highly valued worldwide due to its properties. One of the profitable parts of the tree are the seeds. In the present work the oil extraction was developed using a Soxhlet extractor from Moringa oleifera Lam. Plain variety seeds of Cuban origin. The percentage of extraction was established as response variable in the experimental design. The factors temperature extraction and solute-solvent ratio had a significant influence in the response factor, with probability values lower than 0.05. A mathematical model was obtained which explained the $94.03 \%$ of the variability in the response factor. The best condition for oil extraction was reached operating at a relation solute-solvent $1: 6$ and an extraction temperature of $65^{\circ} \mathrm{C}$.

\section{INTRODUCCIÓN}

Los productos útiles que se han obtenido directa o indirectamente de las plantas han demostrado la importancia que tienen para el hombre. Las plantas se utilizan como fuente de productos medicinales, sirven de fuente de alimento y energía, por lo que es necesario su estudio. En este sentido, las materias primas de origen vegetal se han convertido en un factor primordial y juegan un papel fundamental en el desarrollo de la sociedad actual. Poseen gran aceptación a nivel mundial debido a sus características biodegradables, comestibles y energéticas, lo que permite suplir la explotación de los recursos no renovables que poco a poco se agotan (Díaz et al., 2014). 
En los últimos tiempos se ha incrementado el cultivo de la Moringa oleifera en regiones tropicales y subtropicales, específicamente en Cuba. El estudio de sus semillas tiene gran importancia, pues es una de las especies vegetales con mayor contenido de aceite (30-45\%). El mismo puede tener varios usos reconocidos a nivel internacional, entre ellos están su potencialidad como biocombustible (Mofijur et al., 2014), así como ser un coagulante natural para el tratamiento de aguas residuales y usado en la industria cosmética. En la provincia de La Habana, como en el resto de Cuba, se cuenta con extensos sembrados de Moringa oleifera, que han demostrado buena adaptabilidad a las condiciones agroecológicas existentes en el país.

La creciente demanda en el consumo de aceites vegetales y sus derivados ha provocado la necesidad de importar materias primas para satisfacer los requerimientos de la población. En este sentido, reviste interés la búsqueda y explotación de fuentes oleaginosas nacionales no convencionales dentro de los recursos agroalimentarios con que cuenta el país. Una solución factible a esta problemática está representada en la extracción de aceite de semillas de Moringa oleifera; no obstante, el estudio sobre la extracción de aceite no ha sido totalmente investigado.

El hexano es el solvente más utilizado en el proceso de extracción de aceite. Las razones pueden ser su disponibilidad a un costo razonable, así como su no reactividad con el óleo, y su alta capacidad de disolver ácidos grasos a temperaturas relativamente bajas. Sin embargo, la necesidad de emplear solventes ambientalmente inocuos, menos peligrosos, no inflamables y eficientes, ha seguido siendo una fuerte motivación de interés y búsqueda continua. El etanol podría ser un solvente alternativo debido a su baja toxicidad y su carácter renovable, así se aprovecharía la gran capacidad productiva de etanol a partir de la caña de azúcar con que cuenta Cuba y podría minimizarse el impacto ambiental generado por sustancias tóxicas para estos fines.

El objetivo de este trabajo es determinar las condiciones de operación del proceso de extracción del aceite contenido en las semillas de Moringa oleifera Lam. variedad Plain de origen cubano empleando etanol como solvente.

\section{MATERIALES Y MÉTODOS}

\section{Semillas de Moringa oleifera Lam.}

La variedad de semilla de Moringa oleifera Lam. utilizada es Plain de origen cubano, procedente del Centro Internacional de Salud "La Pradera", localizado en $23^{\circ} 7^{\prime} 0^{\prime \prime} \mathrm{N}, 82^{\circ} 23^{\prime} 0^{\prime \prime} \mathrm{W}$, La Habana, Cuba. Las semillas fueron cosechadas y almacenadas durante tres meses previos a la investigación.

Solvente empleado para la extracción sólido-líquido El solvente empleado es el etanol absoluto (temperatura de ebullición $78.15^{\circ} \mathrm{C}$, gravedad específica 0.789, índice de refracción 1.361).

\section{Preparación de las semillas}

Las semillas se preparan para el proceso de extracción separando primeramente las cáscaras de los cotiledones (descascarado manual) ejerciendo presión sobre las semillas, posteriormente se disminuye el tamaño de partícula triturando con un mortero de laboratorio; de esta manera se garantiza que una vez en el proceso de extracción el área de contacto entre el sólido y el solvente sea mayor, con lo que se logra además favorecer la difusión del solvente en la estructura sólida.

\section{Secado previo al proceso de extracción. Curva de secado}

Al ser trituradas, las semillas son sometidas a un proceso de secado. La determinación se efectúa por diferencia de pesadas, utilizando una balanza analítica Sartorius BS 124S. Para el secado se emplea una estufa, modelo DHG-916A, por espacio de $2 \mathrm{~h}$ a una temperatura de $55^{\circ} \mathrm{C}$. De esta forma, la semilla se encuentra lista para el proceso de extracción. Para determinar la humedad se usa la ecuación 1.

$$
H=\frac{(\text { masa húmeda }- \text { masa seca })}{\text { masa seca }} * 100
$$

Para obtener la curva de secado las semillas se someten al proceso de secado con las condiciones anteriormente mencionadas, pesando muestras cada media hora hasta obtener un peso constante, lo que permite fijar la masa seca para los cálculos de humedad.

\section{Etapa de extracción}

Para el proceso de extracción se emplea el extractor Soxhlet. Preparadas las semillas para cada extracción, se pesa una masa de aproximadamente $10 \mathrm{~g}$ empleando una balanza analítica Sartorius BS 124S. 
IIVESTIGACIÓn Y CIERCIA DE LA UחIVERSIDAD AUTÓnOMH

La cantidad de solvente se adiciona considerando las relaciones soluto-solvente definidas en el diseño de experimentos. En el condensador se utiliza agua como refrigerante. Las temperaturas de extracción se regulan en una plancha eléctrica IKA C-MAG HP10. Para el control de temperatura se emplea un sensor de temperatura modelo Gefran 600 RR001.

\section{Etapa de separación de la mezcla aceite-solvente}

La mezcla aceite-solvente se somete a un proceso de rotoevaporación en un rotoevaporador IKA-WERK (HB 4 basic) para separar el aceite del solvente. La rotoevaporación se efectúa a una temperatura de $50{ }^{\circ} \mathrm{C}$ durante $10 \mathrm{~min}$. Los porcentajes de extracción másico de aceite se calculan mediante la ecuación 2.

$\%$ Extracción de aceite $=\left(\frac{\text { masa aceite }}{\text { masa semilla seca }}\right) * 100$

\section{Estudio cinético}

Antes de realizar el diseño experimental se analiza el comportamiento del porcentaje de extracción en función del tiempo y se procede a fijar el tiempo de extracción para todos los casos, por lo que esta variable se considera independiente en el diseño de experimentos. Este procedimiento se realiza a la relación soluto-solvente 1:6.

\section{Diseño experimental}

Para el diseño de experimentos se trabaja con el programa estadístico Statgraphics Centurion versión $\mathrm{XV}$. Se propone un diseño factorial $2^{2}$, considerando dos factores a dos niveles: los factores considerados son la temperatura de extracción y la relación soluto-solvente, los niveles de estudio para el factor temperatura de extracción son 78 y $65^{\circ} \mathrm{C}$, y para la relación soluto-solvente, 1:4 y 1:6. Estas son relaciones masa-volumen; es decir, masa de semilla entre volumen de solvente. En todos los casos, la variable dependiente es el porcentaje de extracción de

Tabla 1

Niveles de variación de los factores

\begin{tabular}{ccc}
\hline Factor & Valor real & $\begin{array}{c}\text { Valor } \\
\text { codificado }\end{array}$ \\
\hline Temperatura de extracción & 78 & 1 \\
$\left({ }^{\circ} \mathrm{C}\right)$ & 65 & -1 \\
\hline Relación soluto-solvente (g de & $1-6$ & 1 \\
semilla/volumen de solvente) & $1-4$ & -1 \\
\hline
\end{tabular}

Nota: Elaboración propia. aceite. Para todos los experimentos se hacen dos réplicas para un total de 12 experimentos. En la tabla 1 se muestran los valores reales y codificados de cada factor en estudio.

RESULTADOS

\section{Preparación de las semillas. Secado}

Al someter las semillas al proceso de secado durante 2 h a $55^{\circ} \mathrm{C}$ se obtiene una humedad promedio de $4.82 \%$ \pm 0.03 . Para la realización de la curva de secado se obtiene una masa de semilla seca de $14.49 \mathrm{~g}$, la cual se emplea para el cálculo de humedad en función del tiempo. La curva de secado de la variedad en cuestión (Plain) se compara con las curvas paras las variedades cubanas Criolla y Supergenius con el objetivo de conocer el porcentaje de humedad previo al proceso de extracción (figura 1).

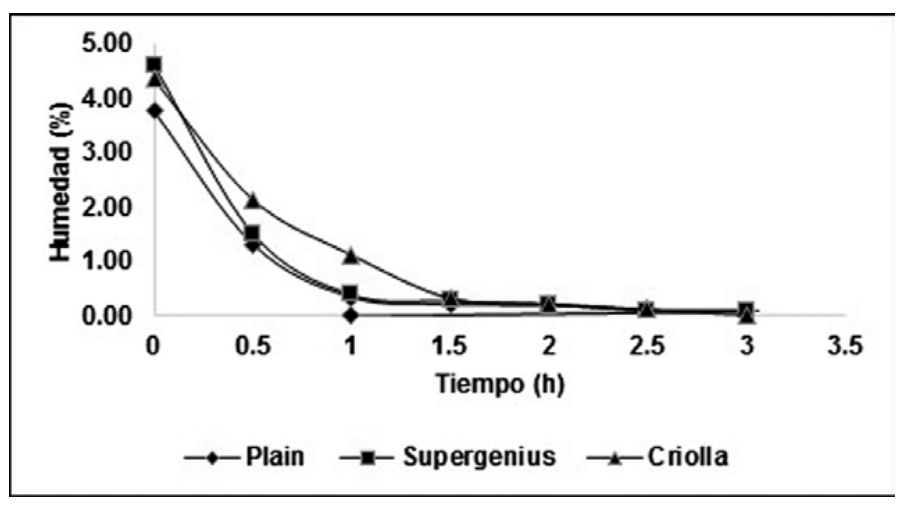

Figura 1. Curva de secado.

Elaboración propia.

Al ser desconocida la cinética del proceso de extracción para la variedad Moringa oleifera Plain de origen cubano, se analiza el comportamiento del porcentaje de extracción de aceite para la relación soluto-solvente 1:6 a diferentes tiempos. El estudio cinético se realiza con la finalidad de realizar las extracciones a un tiempo constante $y$, de esta manera, fijar este parámetro para todas las extracciones. En la figura 2 se muestra la curva obtenida.

\section{Extracción de aceite. Diseño experimental}

La variable fundamental que se evalúa es el porcentaje de extracción de aceite y para esto se definen como factores influyentes la temperatura de extracción y la relación soluto-solvente. En la tabla 2 se definen los porcentajes de extracción. 


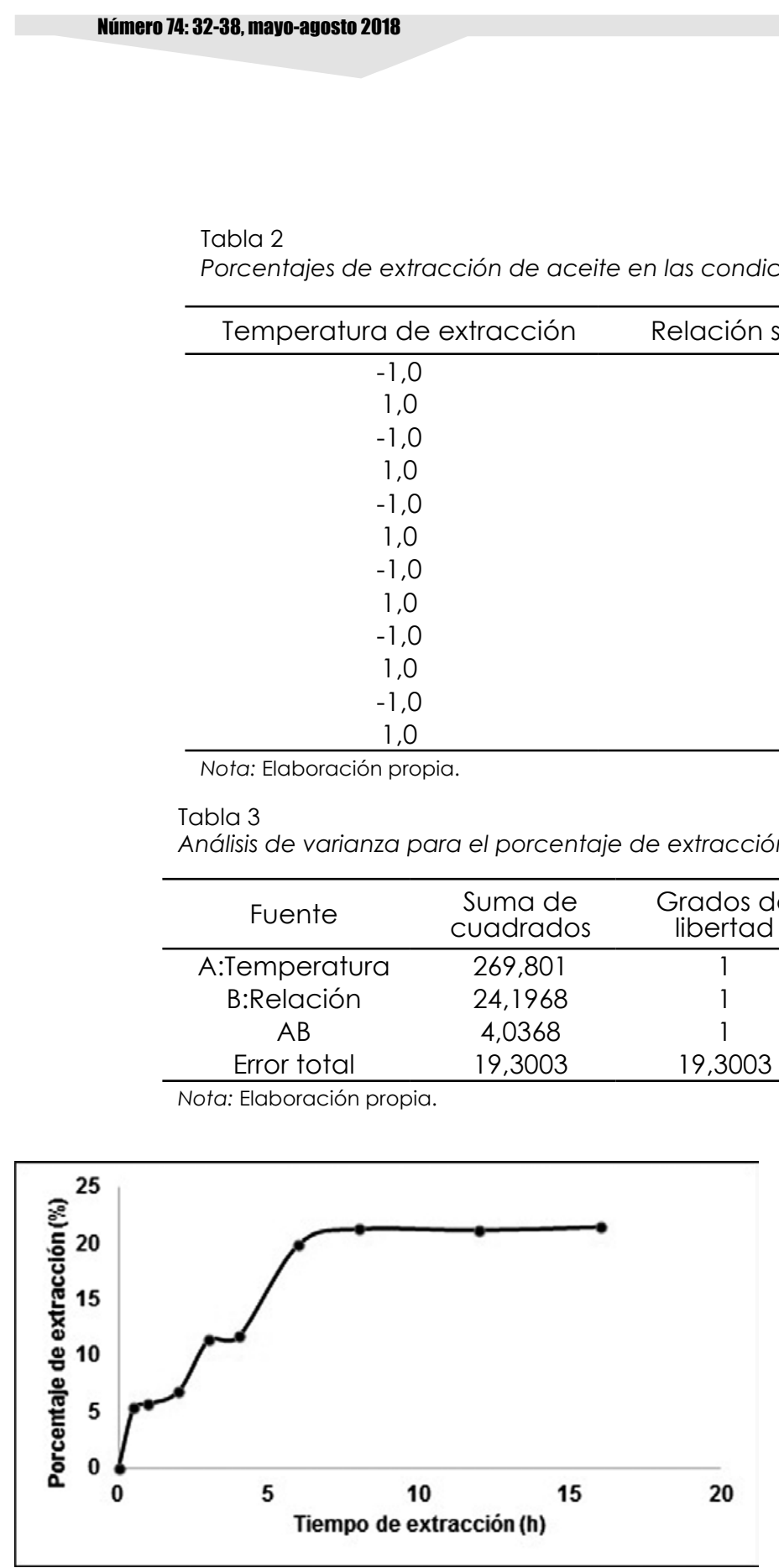

Figura 2. Influencia del tiempo de reacción en el porcentaje de extracción de aceite.

Elaboración propia.

\section{Análisis estadístico del diseño experimental}

El diseño experimental que se propone es un diseño factorial $2^{2}$ con dos réplicas, y su procesamiento permite la obtención de un modelo que explica el comportamiento de la extracción de aceite de semillas de Moringa oleifera Lam., teniendo en cuenta las condiciones experimentales estudiadas. El modelo en variables codificadas que se obtiene se representa por la ecuación 3.

$$
E=22,6517-4,74167 * T+1,42 * R+0,58 * T * R
$$

Tabla 2
IIVESTIGACIÓn Y CIERCIA DE LA UחIVERSIDAD AUTÓnOMA DE RGUASCALIEחTES

\begin{tabular}{cc} 
Soluto-solvente & Extracción de aceite (\%) \\
\hline 1,0 & 27,85 \\
1,0 & 16,64 \\
$-1,0$ & 25,81 \\
1,0 & 21,81 \\
1,0 & 29,90 \\
1,0 & 14,25 \\
1,0 & 28,98 \\
1,0 & 19,91 \\
1,0 & 26,95 \\
1,0 & 16,84 \\
1,0 & 24,87 \\
1,0 & 18,01 \\
\hline
\end{tabular}


A partir del análisis de varianza para la variable respuesta Porcentaje de extracción de aceite se obtiene el valor del coeficiente Durbin-Watson. El estado deseado se encuentra en el entorno de dos, y esto ocurre cuando la probabilidad es mayor que 0.05. En el diseño de experimentos que se analiza tiene un valor de 2.90884 asociado a una probabilidad de 0.8557. De esta manera, se puede afirmar que no existe correlación alguna en los errores, o lo que es equivalente, hay independencia entre los mismos. Para las condiciones estudiadas el mejor valor tomado como criterio para maximizar el porcentaje de aceite extraído es de $28.23 \%$ para una relación soluto-solvente 1:6 y una temperatura de $65^{\circ} \mathrm{C}$.

\section{DISCUSIÓN}

\section{Preparación de las semillas. Secado}

En la figura 1 se observa que en $2 \mathrm{~h}$ las semillas de las variedades Plain, Criolla y Supergenius poseen una humedad de $0.20,0.23$ y $0.22 \%$, respectivamente, lo que demuestra la similitud en estos valores y que la condición establecida de $2 \mathrm{~h}$ a $55^{\circ} \mathrm{C}$ en el secado es efectiva y garantiza un bajo porcentaje de humedad de modo previo al proceso de extracción de aceite. Anwar y Bhanger (2003) extrajeron aceite de semillas de Moringa oleifera cultivadas en 12 ciudades de Pakistán, las mismas fueron colectadas durante los meses de julio y agosto. La humedad determinada a estas semillas evidenció un valor promedio de $5.70 \pm 0.42$. Igualmente, Ortiz Palafox et al. (2012) reportaron para semillas de la península de Yucatán una humedad de $5.84 \%$.

Si se realiza una comparación con los resultados obtenidos en esta investigación, la humedad en las semillas de Moringa oleifera variedad Plain es inferior. Sin embargo, no se reportan las condiciones a las que se determina la humedad en las variedades de Pakistán y Yucatán, y las diferencias pueden estar relacionadas con la calidad de las semillas utilizadas, desde su cosecha hasta el almacenamiento. Igualmente, para las variedades cubanas Criolla y Supergenius se obtienen humedades de 4.36 y $4.61 \%$, respectivamente. Es importante tener en cuenta que los porcentajes de humedad reportados en la literatura son los iniciales, y no se conoce con exactitud la humedad de la muestra para su posterior tratamiento.

\section{Estudio cinético}

En la figura 2 se evidencia un comportamiento exponencial, lo que indica un incremento en la extracción del aceite en el tiempo; sin embargo, existe un valor de tiempo a partir del cual la cantidad de aceite que se extrae es prácticamente constante, lo que se evidencia por un comportamiento asintótico de las curvas. El tiempo de extracción que se selecciona es de $6 \mathrm{~h}$, con ello se garantiza extraer cerca de la zona donde comienza el comportamiento asintótico.

\section{Extracción de aceite. Diseño experimental}

La tabla 2 muestra que los porcentajes de extracción varían en un intervalo entre 14 y $30 \%$. Los resultados revelan que los mayores porcentajes de extracción se obtienen a una temperatura de extracción de 65 ${ }^{\circ} \mathrm{C}$ y una relación soluto-solvente 1:6. La influencia de la relación soluto-solvente viene dada que a mayor relación soluto-solvente mayor porcentaje de extracción, ya que debido a la polaridad y volatilidad del etanol hay que extraer con volúmenes más grandes para asegurar una correcta operación, y la no existencia de explosión al quedar seco el balón.

Con respecto a la temperatura se conoce que un aumento de la temperatura favorece la solubilidad del soluto en el solvente y aumenta el coeficiente de difusividad en las partículas del sólido. Lo antes explicado demuestra que al aumentar la temperatura aumenta el porcentaje de extracción de aceite. La causa de una proporcionalidad inversa entre la temperatura y el porcentaje de extracción en este estudio viene dado por posibles reacciones químicas paralelas (reacción de transesterificación) que se presentan cuando se trabaja a temperaturas superiores a $70{ }^{\circ} \mathrm{C}$ entre el aceite y el etanol, lo que disminuye el porcentaje de extracción y propicia la coloración oscura del producto extraído. Este planteamiento es demostrado por Efeovbokhan, Hymore, Raji y Sanni (2015), quienes reportaron dicho comportamiento.

Abdulkareem, Uthman, Afolabi y Awenebe (2011) reportaron porcentajes entre $20-30 \%$ empleando etanol para semillas colectadas en el estado de Niger, Nigeria. Ortiz Palafox et al. (2012) empleando el mismo solvente obtuvieron $25 \%$ de extracción en semillas molidas. Al comparar los resultados obtenidos en la presente investigación con los citados anteriormente, se evidencia que existe correspondencia. Lalas y Tsaknis (2002) reportaron para la variedad Periyakulam 1 de la India un porcentaje de 38.3\%, mientras que Latif y Anwar (2008) 38.40\% 


\section{IIVESTIGAGIÓn Y CUERCIA DE LA UחIVERSIDAD AUTÓNOMA DE AGUASCALIERTES}

con semillas de la variedad Concanesis de Pakistán, y Garba, Medugu, Gwaski y Amusat (2015) 39\% de extracción en semillas molidas del estado de Borno, Nigeria.

Adegbe, Larayetan y Omojuwa (2016) obtuvieron $38 \%$ de extracción con semillas colectadas en el oeste del estado de Kwara, Efeovbokhan et al. (2015) alcanzaron $34.71 \%$ de semillas provenientes del estado de Oyo, ambas de Nigeria, y Oliveira et al. (2012) $40 \%$ de semillas colectadas en el estado de Río Grande, Brasil. También Adejumo, Alakowe y Obi (2013) mediante semillas originarias de KureMarket en el estado de Niger, Nigeria, logran obtener un $33.7 \%$, y Goja (2013) 34.5\% empleando semillas provenientes de Ed Deium, Sudán. Las extracciones de dichos trabajos se llevaron a cabo utilizando hexano como solvente. Mani, Jaya y Vadivambal (2007) alcanzaron un máximo porcentaje de $31.8 \%$ empleando éter de petróleo en semillas de la variedad Periyakulam 1. Efeovbokhan et al. (2015) emplearon éter de petróleo para extraer semillas provenientes del estado Kaduna y Oyo, del norte y sureste de Nigeria, y obtuvieron un porcentaje promedio de $37.57 \%$.

Es de notar que los porcentajes de extracción obtenidos con etanol son menores con respecto a los alcanzados con hexano y éter de petróleo. La limitación está dada por la polaridad del etanol. El aceite que se extrae está formado por moléculas apolares, polaridad similar a la del hexano y el éter de petróleo; sin embargo, el etanol está formado por moléculas polares. Es importante destacar que el empleo del etanol absoluto garantiza una baja toxicidad, menor costo de adquisición y mayor disponibilidad, lo que evidencia su aplicabilidad. En las figuras 3 y 4 se muestran las dos muestras de aceite obtenido a las dos temperaturas en estudio (65 y 78 $\left.{ }^{\circ} \mathrm{C}\right)$, respectivamente.

En la figura 3 es evidente la diferencia en el color del aceite obtenido. Esto es debido a la reacción de transesterificación que propicia la obtención de glicerina como subproducto (Efeovbokhan et al.,

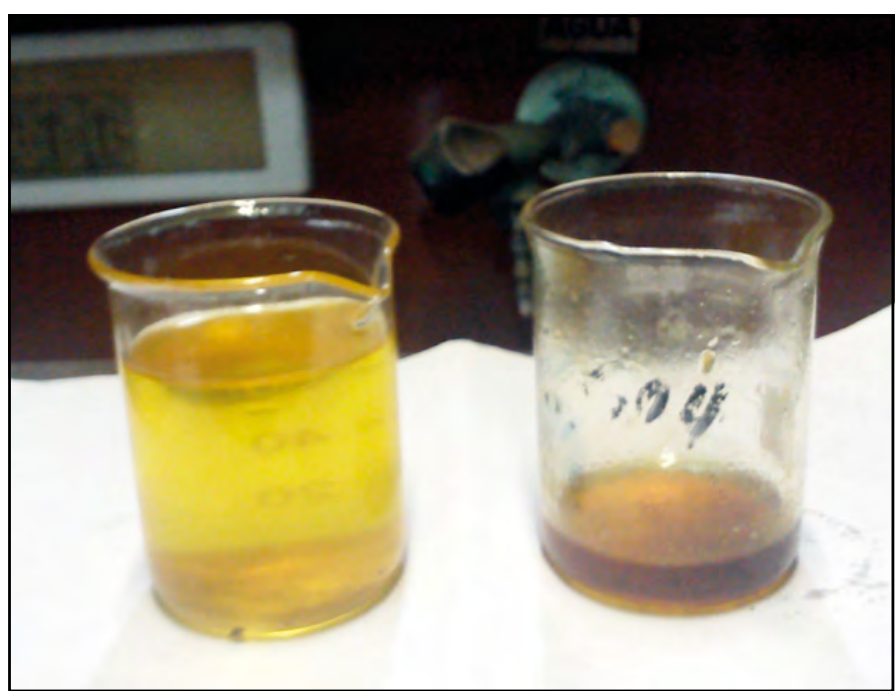

Figura 3. Aceite extraído a 65 y $78^{\circ} \mathrm{C}$, respectivamente. Imagen del equipo de investigación.

2015), cuando se extrae a una temperatura mayor que $70^{\circ} \mathrm{C}$. Por esta razón es favorable extraer a 65 ${ }^{\circ} \mathrm{C}$, alcanzando mayores porcentajes de extracción y evitando la coloración oscura del aceite.

\section{CONCLUSIONES}

Se demuestra que para las condiciones estudiadas la extracción de aceite de las semillas de Moringa oleifera variedad Plain de origen cubano logra un porcentaje de extracción de $29.90 \%$. Los factores temperatura de extracción y relación solutosolvente influyen significativamente en el proceso de extracción de aceite. El modelo obtenido a partir del diseño de experimentos establecido explica $94.03 \%$ de la variabilidad del porcentaje de extracción de aceite. El mayor porcentaje se obtuvo a $6 \mathrm{~h}$, una relación soluto-solvente 1:6 y a $65^{\circ} \mathrm{C}$. Se comprueba que en las extracciones realizadas a temperaturas superiores a $70{ }^{\circ} \mathrm{C}$ se produce una disminución del porcentaje de extracción y un cambio en la coloración del aceite propiciado por reacciones químicas paralelas, lo que no ocurrió así a $65^{\circ} \mathrm{C}$. 


\section{LA UNIVERSIDAD AUTÓNOMA DE AGUASCALIERTES}

REFERENCIAS

- Abdulkareem, A. S., Uthman, H., Afolabi, A. S., \& Awenebe, O. L. (2011). Extraction and optimization of oil from Moringa oleifera seed as alternative feedstock for the production of biodiesel. En M. Nayeripour, \& M. Kheshti (Eds.), Sustainable growth and applications in renewable energy sources (cap. 12, pp. 244-268). Croatia-China: InTech. doi: 10.5772/25855

- Adegbe, A. A., Larayetan, R. A., \& Omojuwa, T. J. (2016). Proximate analysis, physicochemical properties and chemical constituents characterization of Moringa oleifera (Moringaceae) seed oil using GC-MS analysis. American Journal of Chemistry, 6(2), 23-28.

- Adejumo, B. A., Alakowe, A. T., \& Obi, D. E. (2013). Effect of heat treatment on the characteristics and oil yield of Moringa oleifera seeds. The International Journal of Engineering And Science (IJES), 2(1), 232-239.

- Anwar, F., \& Bhanger, M. I. (2003). Analytical characterization of Moringa oleifera seed oil grown in temperate regions of Pakistan. Agricultural and Food Chemistry, 51 (22), 6558-6563.

- Anwar, F., \& Rashid, U. (2007). Physico-chemical characteristics of Moringa oleifera seeds and seed oil from a wild provenance of Pakistan. Pakistan Journal of Botany, 39(5), 1443-1453.

- Díaz, Y., Rodríguez, S., Ferrer, C., Mazorra, M., Fernández, E., \& Zumalacárregui, B. (2014). Biocombustibles para su uso en motores Diesel. La Habana: IDICT.

- Efeovbokhan, V. E., Hymore, F. K., Raji, D., \& Sanni, S. E. (2015). Alternative solvents for Moringa oleifera seeds extraction. Journal of Applied Sciences, 15(8), 1073-1082.

- Garba, A. A., Medugu, D. W., Gwaski, P. A., \& Amusat, R. O. (2015). Extraction and characterization of Moringa oleifera seed oil. Applied Research Journal, 1 (9), 473-477.
- Goja, A. M. (2013). Physico-chemical properties of oil produced from Moringa oleifera, Jatropha curcas and Carthamus tinctorius L. seeds. International Journal of Advanced Research, 1(4), 181-187.

- Lalas, S., \& Tsaknis, J. (2002). Characterization of Moringa oleifera seed oil variety "Periyakulam 1". Journal of Food Composition and Analysis, 15(1), 65-77.

- Latif, S., \& Anwar, F. (2008). Quality assessment of Moringa concanensis seed oil extracted through solvent and aqueousenzymatic techniques. Grasas y Aceites, 59(1), 69-75.

- Mani, S., Jaya, S., \& Vadivambal, R. (2007). Optimization of solvent extraction of Moringa oleifera seed kernel oil using response surface methodology. Food and Bioproducts Processing, 85(C4), 328-335.

- Mofijur, M., Masjuki, H. H., Kalam, M. A., Atabani, A. E., Arbab, M. I., Cheng, S. F., \& Gouk, S. W. (2014).Properties and uses of Moringa oleifera biodiesel and diesel blends in a multi-cylinder diesel engine. Energy Conversion and Management, 82, 169176.

- Oliveira, D. S., Fonseca, X. D. S., Farias, P. N., Bezerra, V. S., Pinto, C. H. C., Souza, L. D., ..., \& Matias, L. G. O. (2012). Obtenção do biodiesel a través do transesterificação do óleo de Moringa oleífera Lam. HOLOS, 1, 49-61.

- Ortiz Palafox, J., Navarrete, A., Sacramento-Rivero, J. C., RubioAtoche, C., Acereto Escoffie, P., \& Rocha-Uribe, J. A. (2012). Extraction and characterization of oil from Moringa oleifera using supercritical $\mathrm{CO}_{2}$ and traditional solvents. American Journal of Analytical Chemistry, 3, 946-949. 